\title{
Stable state of Mode A for flow past a circular cylinder
}

\author{
HONGYI JIANG ${ }^{1 \dagger}$, LIANG CHENG ${ }^{1,2}$, FEIFEI TONG ${ }^{1}$, SCOTT DRAPER $^{1,3}$ AND \\ HONGWEI AN ${ }^{1}$ \\ ${ }^{1}$ School of Civil, Environmental and Mining Engineering, The University of Western Australia, 35 \\ Stirling Highway, Crawley, WA 6009, Australia \\ ${ }^{2}$ State Key Laboratory of Coastal and Offshore Engineering, Dalian University of Technology, \\ Dalian, 116024, China \\ ${ }^{3}$ Centre for Offshore Foundation Systems, The University of Western Australia, 35 Stirling \\ Highway, Crawley, WA 6009, Australia
}

\begin{abstract}
A stable three-dimensional (3D) wake structure for flow past a circular cylinder has been discovered through 3D direct numerical simulations (DNS). The stable 3D wake structure occurs over a small range of Reynolds number $(R e)$ below the critical Re for Mode A* (i.e. Mode A with large-scale vortex dislocations, where Mode A is the first $3 \mathrm{D}$ instability mode which will evolve into Mode $\mathrm{A}^{*}$ ) instability. It is believed that the stable 3D wake structure discovered in this study is the stable state of Mode A wake structure inferred by Williamson (J. Fluid Mech. vol. 328, 1996, pp. 345-407). This confirms the wake transition sequence of $2 \mathrm{D} \rightarrow \mathrm{A} \rightarrow \mathrm{A}^{*} \rightarrow \mathrm{B}$ suggested by Williamson (1996b). Compared with conventional Mode A structure, the stable state of Mode A structure has much weaker amplitude and does not evolve into large-scale vortex dislocations. The stable state of Mode A structure is triggered by small-scale spanwise disturbance introduced upstream of the cylinder, due to energy amplification through convective instability of the flow. The stable state of Mode A is transient and is damped out eventually under a transient initial disturbance condition, but is sustained throughout under a persistent disturbance condition. The emergence of stable state of Mode A structure is correlated with both $R e$ and the disturbance level. With the decrease of $R e$, the stable state of Mode A structure gradually becomes less
\end{abstract}

\footnotetext{
†Email address for correspondence: hongyijiang88@gmail.com
} 
well-defined and eventually disappears. With the decrease of the disturbance level, the stable state of Mode A structure emerges at a higher $R e$.

\section{Introduction}

Flow past a long circular cylinder at relatively low values of the Reynolds number $(R e)$ has been the topic of extensive studies due to its fundamental and practical significance. It is well known that the flow is governed by a single dimensionless parameter $R e$, which is defined based on the approaching flow velocity $U$, the cylinder diameter $D$ and the kinematic viscosity of the fluid $v$. Methods of investigation of flow past cylinders include physical model testing, direct numerical simulation (DNS) and linear (and non-linear) stability analysis. Comprehensive reviews on investigation of flow past a circular cylinder with different methodologies can be found, for example, in Williamson (1996a, 1996b) and Posdziech and Grundmann (2001). It has been well understood (e.g. Williamson, 1996a) that with increasing $R e$ the flow structure in the cylinder wake undergoes a transition sequence of: (1) emergence of two-dimensional (2D) primary wake instability at $R e \sim 47$, (2) onset of (conventional) Mode A (the first three-dimensional (3D) instability mode, with a spanwise wavelength of approximately $4 D$ and an out-of-phase sequence between the neighbouring braids) instability with large-scale vortex dislocations (denoted as Mode $\mathrm{A}^{*}$ ) at $R e \sim 190$, (3) gradual transition from Mode $\mathrm{A}^{*}$ to Mode $\mathrm{B}$ (the second $3 \mathrm{D}$ instability mode, with a spanwise wavelength of approximately $0.82 \mathrm{D}$ and an in-phase sequence between the neighbouring braids) over a range of $R e$ from 230 to 250, and (4) development of increasingly disordered Mode B structure for $R e$ beyond 260. The above transition sequence of $2 \mathrm{D} \rightarrow \mathrm{A}^{*} \rightarrow \mathrm{B}$ (Williamson, 1996b) is routinely referred to in the literature. However, Williamson (1996b) inferred that "there exists a very small range of $R e$ for which the flow is unstable to small scales of Mode A, but whose amplitude is too weak to trigger intermittent vortex dislocations". Based on this, Williamson (1996b) suggested a possible flow transition sequence of $2 \mathrm{D} \rightarrow \mathrm{A} \rightarrow \mathrm{A}^{*} \rightarrow \mathrm{B}$, where mode " $\mathrm{A}$ " is free from vortex dislocations. This transition 
sequence is not widely adopted in the literature mainly because the stable state of Mode A has not been confirmed either experimentally or numerically.

Many previous studies have focused on identifying the critical Reynolds number $R e_{c r}$ at which point Mode A* wake instability develops. The $R e_{c r}$ values identified by some of experimental studies are: 150 by Roshko (1954) and Tritton (1959), 165 by Norberg (1994), 178 by Williamson (1988, 1989), 194 by Williamson (1996b), and 205 by Miller and Williamson (1994). It was discovered by Williamson (1996b) that the low values of $R e_{c r}$ identified by early experimental studies were largely due to the “end effect". Williamson (1996b) extended the $R e_{c r}$ value to 194 by eliminating end effects using non-mechanical end conditions. This value is very close to the $R e_{c r}$ values of $188.5( \pm 1.0), 190.2( \pm 0.02)$, and 190.5, predicted through linear stability analysis by Barkley and Henderson (1996), Posdziech and Grundmann (2001), and Rao et al. (2013), respectively. In contrast to linear stability analysis, many previous non-linear 3D DNS studies reported higher $R e_{c r}$ values of $\sim 200$ (e.g. Karniadakis and Triantafyllou, 1992; Behara and Mittal, 2010; Zhao et al., 2013; Tong et al., 2014). However, based on a further refined computational mesh, the $R e_{c r}$ predicted by Jiang et al. (2016) was reduced to $193-194$.

In previous 3D DNS studies, the $R e_{c r}$ was normally identified by observing a corresponding sudden drop of the Strouhal number $S t$ in the $S t-R e$ relationship (e.g. Behara and Mittal, 2010; Zhao et al., 2013; Tong et al., 2014; Jiang et al., 2016) or by analysing the growing/decaying trend of the time-history of spanwise velocity (e.g. Karniadakis and Triantafyllou, 1992). However, less attention has been paid to the wake flow structure at $R e<R e_{c r}$, although the wake structure for $R e>R e_{c r}$ has been studied extensively. Hence to the best knowledge of the authors, the stable state of Mode A structure at $R e<R e_{c r}$ inferred by Williamson (1996b) has neither been confirmed nor been proven non-existent by previous 3D DNS studies reported in the literature.

The primary aim of this study is therefore to examine and explain the existence of the stable state of Mode A structure at $R e<R e_{c r}$ by using 3D DNS. To work towards this aim, the rest of this paper is organized in the following manner. In $\S 2$, the 
governing equations and numerical model are presented. The numerical results are then discussed in $\S 3$. Finally, major conclusions are drawn in $\S 4$.

\section{Numerical model}

\subsection{Numerical method}

The DNS have been carried out with OpenFOAM (www.openfoam.org) through solution of the continuity equation and the incompressible Navier-Stokes equations:

$$
\begin{aligned}
& \frac{\partial u_{i}}{\partial x_{i}}=0 \\
& \frac{\partial u_{i}}{\partial t}+u_{j} \frac{\partial u_{i}}{\partial x_{j}}=-\frac{1}{\rho} \frac{\partial p}{\partial x_{i}}+v \frac{\partial^{2} u_{i}}{\partial x_{j} \partial x_{j}}
\end{aligned}
$$

where $\left(x_{1}, x_{2}, x_{3}\right)=(x, y, z)$ are Cartesian coordinates, $u_{i}$ is the velocity component in the direction $x_{i}, t$ is time and $p$ is pressure. Equations (2.1) and (2.2) are solved with the finite volume method (FVM) and the PISO (Pressure Implicit with Splitting of Operators) algorithm (Issa, 1986). The convection term is discretized using the

fourth-order cubic scheme, while the diffusion term is discretized using a second-order linear scheme. A blended scheme consisting of the second-order Crank-Nicolson scheme and a first-order Euler implicit scheme is used to integrate the equations in time.

\subsection{Computational domain and mesh}

The computational domain and mesh are determined based on a domain size and mesh resolution dependence study for the simulation of flow past a circular cylinder at $R e=300$ reported separately in Jiang et al. (2016). The standard 3D mesh reported in Jiang et al. (2016) is adopted in this study, where the $R e$ values are less than 200. Based on the standard 3D mesh, Jiang et al. (2016) obtained DNS results of the wake transition of flow past a circular cylinder which are in good agreement with the experimental results reported by Williamson (1996a, 1996b). 
For the standard 3D mesh (Jiang et al., 2016), a hexahedral computational domain of $50 D \times 40 D \times 12 D$ as shown in Fig. 1(a) is adopted. In the plane perpendicular to the cylinder span (i.e. the $x-y$ plane), the cylinder perimeter is equally discretized with 132 nodes, and the radial size of the first layer of mesh next to the cylinder is $0.001 D$. The cell expansion ratio in the whole domain is kept below 1.1. For the sake of wake flow visualization, a higher mesh resolution in the near wake is used by specifying a constant cell size along the $x$-direction for $x / D$ ranges from approximately 1.0 to 5.5 . A close-up view of the standard mesh in the $x-y$ plane near the cylinder is shown in Fig. 1(b). The standard $3 \mathrm{D}$ mesh is formed by replicating the $2 \mathrm{D}$ mesh along the $z$-axis, resulting in an identical mesh resolution in all planes perpendicular to the cylinder span. The cell size in the spanwise direction is $0.1 D$, which results in a total of 120 identical layers of mesh along the cylinder span.

For DNS with high accuracy schemes such as spectral element method, flow three-dimensionality is normally initiated by introducing small-scale disturbances to the inlet velocity or the entire flow field at the beginning of the simulation (e.g. Henderson, 1997; Thompson et al., 2001). The amplitudes of the initial disturbance in Henderson (1997) and Thompson et al. (2001) by using the spectral element method were $5 \times 10^{-3} U$ and $1 \times 10^{-4} U$, respectively. In contrast, for conventional DNS based on FVM or finite element method (FEM) formulations, additional artificial disturbances are not normally required because numerical disturbances embedded in the computational domain (e.g. by skewed mesh elements) can automatically trigger flow three-dimensionality. Our previous DNS study (Jiang et al., 2016) based on the same FVM formulation showed that for the standard 3D mesh the amplitude of the spanwise disturbance velocity in the computational domain is approximately $5 \times 10^{-4} \mathrm{U}$ (which is mainly concentrated at the locations where mesh skewness takes place). The $R e_{c r}$ value predicted based on the standard 3D mesh is $193-194$ (Jiang et al., 2016), which is very close to the experimental result of $R e_{c r}=194$ by Williamson (1996b) but slightly $(\sim 2 \%)$ larger than the linear stability analysis results of $188.5( \pm 1.0)$, 190.2 ( \pm 0.02$)$, and 190.5 predicted by Barkley and Henderson (1996), Posdziech and Grundmann (2001), and Rao et al. (2013), respectively, due to the slight influence of 
the mesh resolution. Nevertheless, the standard 3D mesh is still employed in the present study as a compromise between the computational cost and numerical accuracy.

In addition to the standard 3D mesh, a modified 3D mesh with a reduced disturbance level is also employed in the present study. As shown in Fig. 1(c), the modified mesh adopts an enlarged concentric circle of $12 \mathrm{D}$ around the cylinder in the $x-y$ plane (compared with a concentric circle of $1.25 D$ for the standard mesh), while the node number and mesh size next to the cylinder and the size of the computational domain remain unchanged. By using the modified mesh, the amplitude of the spanwise disturbance velocity due to the skewed mesh elements reduces to around $2 \times 10^{-5} U$, which is approximately $1 / 25$ of that induced by the standard 3D mesh.

Through examining the growing/decaying trend of the time-histories of spanwise velocity in the near wake, the $R e_{c r}$ based on the modified 3D mesh is found to be 192.0, which is quite close to the result of 193.3 obtained with the standard 3D mesh. The slight difference is attributed to the slight change of the mesh resolution close to the cylinder in the $x-y$ plane due to the use of a different mesh topology (Fig. 1(b) versus Fig. 1(c)), rather than the introduction of different disturbance levels. This point will be demonstrated later on in $\S 3.5$.

For $R e>R e_{c r}$, since the spanwise velocity will grow into a much larger level (of the order of $U$ ) after the flow is fully developed, the disturbance amplitude of $5 \times 10^{-4} U$ or $2 \times 10^{-5} U$ introduced in the present study will not influence the simulation results noticeably. For example, at $R e=194$ which is just above the $R e_{c r}$, less than $1 \%$ difference of the Strouhal number, mean drag force coefficient, and root-mean-square lift force coefficient for the saturated flow (for a statistical range of 800 non-dimensional time units) is obtained by using the standard and modified 3D meshes. 
(a)

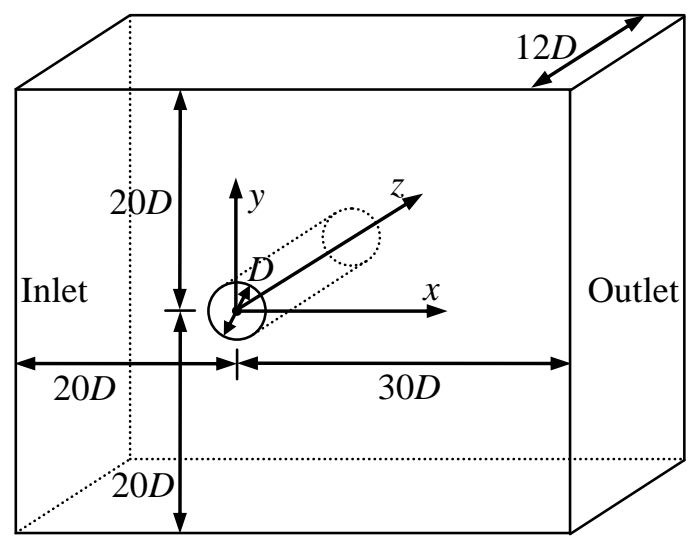

(b)

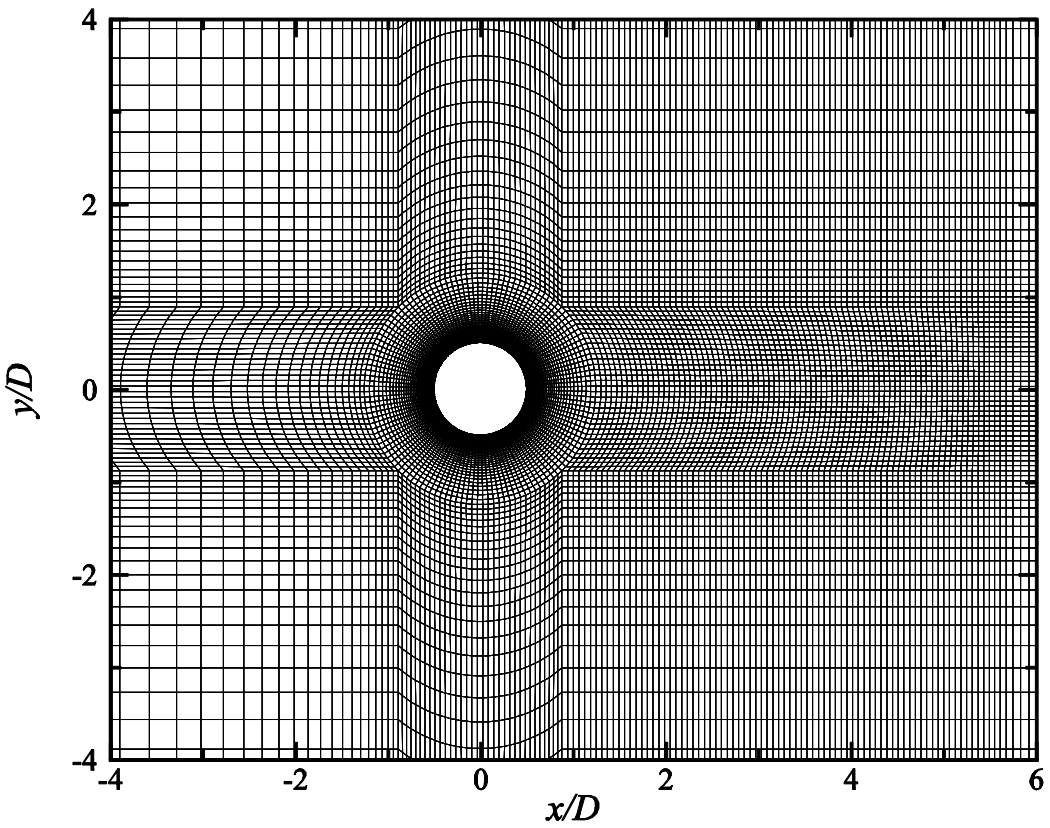

(c)

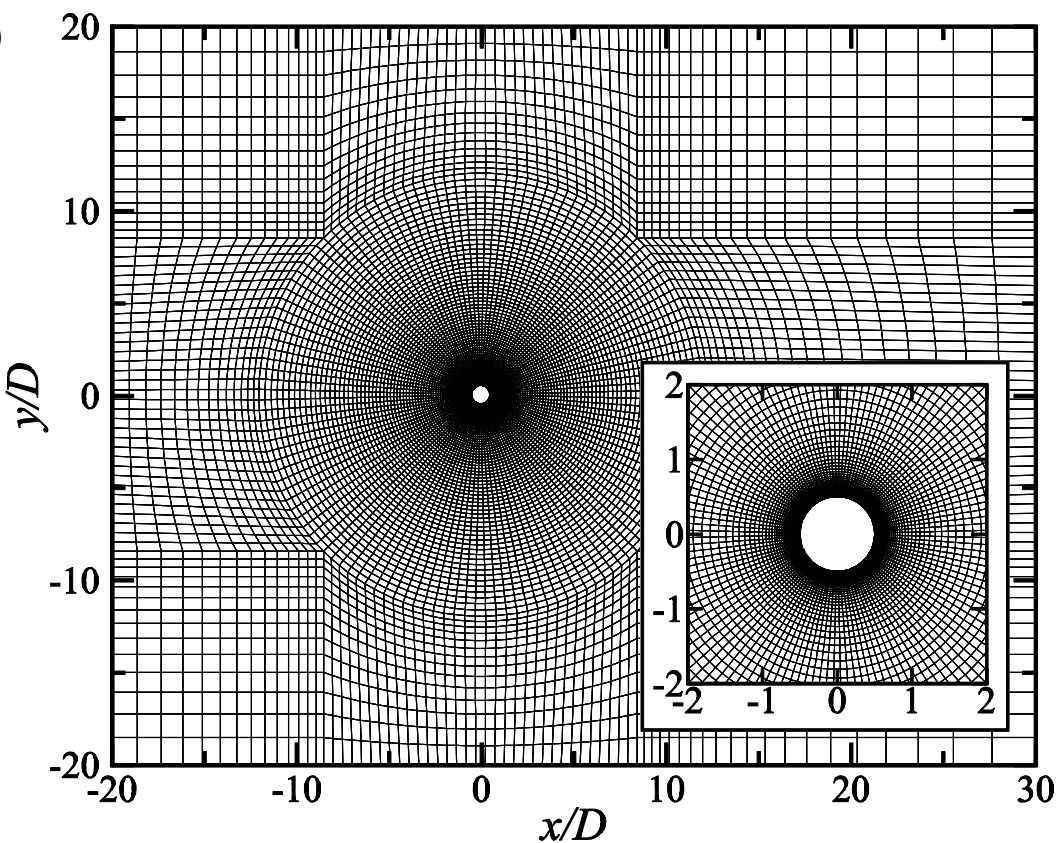

Fig. 1. (a) Schematic model of the computational domain, (b) Close-up view of the standard mesh in the $x-y$ plane near the cylinder, and (c) the modified mesh in the $x-y$ 
plane.

\subsection{Boundary conditions}

The boundary conditions adopted in the present study are the same as those used in Jiang et al. (2016). At the inlet boundary, a uniform flow velocity $U$ is specified in the $x$-direction. At the outlet, the Neumann boundary condition (i.e. zero normal gradient) is applied for the velocity, and the pressure is specified as a reference value of zero. A symmetry boundary condition is applied at the top and bottom boundaries, whereas a periodic boundary condition is employed at the two lateral boundaries perpendicular to the spanwise direction. A non-slip boundary condition is applied on the cylinder surface.

\section{Numerical results}

\subsection{Level of flow three-dimensionality}

The flow three-dimensionality for $R e<R e_{c r}$ is quantified by the spanwise disturbance energy $E_{z}$ integrated over the near wake region of $x / D=0-5$ (with the whole lengths in $y$ - and $z$-directions), where $E_{z}$ is defined as:

$E_{z}=\frac{1}{2} \int_{V}\left(u_{z} / U\right)^{2} \mathrm{~d} V$

and $V$ is the volume of the flow field of interest. Fig. 2(a) shows the time evolution of $E_{z}$ for a few cases calculated with the standard 3D mesh, where $t^{*}(=U t / D)$ denotes the non-dimensional flow time. Each case is calculated to the state where the flow is fully developed. For $R e<R e_{c r}$, the fully developed state is reached when the time-history of the spanwise velocity sampled at a near-wake point becomes periodic with a constant amplitude for every vortex shedding period. For example, according to the time-history of the spanwise velocity sampled at $(x / D, y / D, z / D)=(3,0.5,6)$ for $R e=175$ shown in Fig. 2(b), the flow saturates at $t^{*} \sim 250$. 
(a)

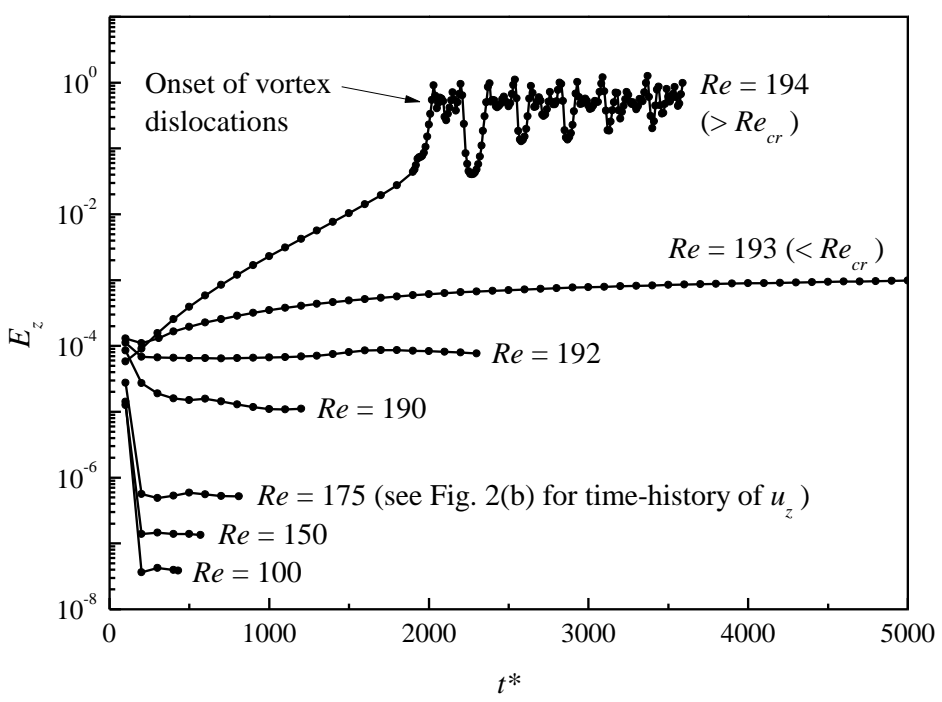

(b)

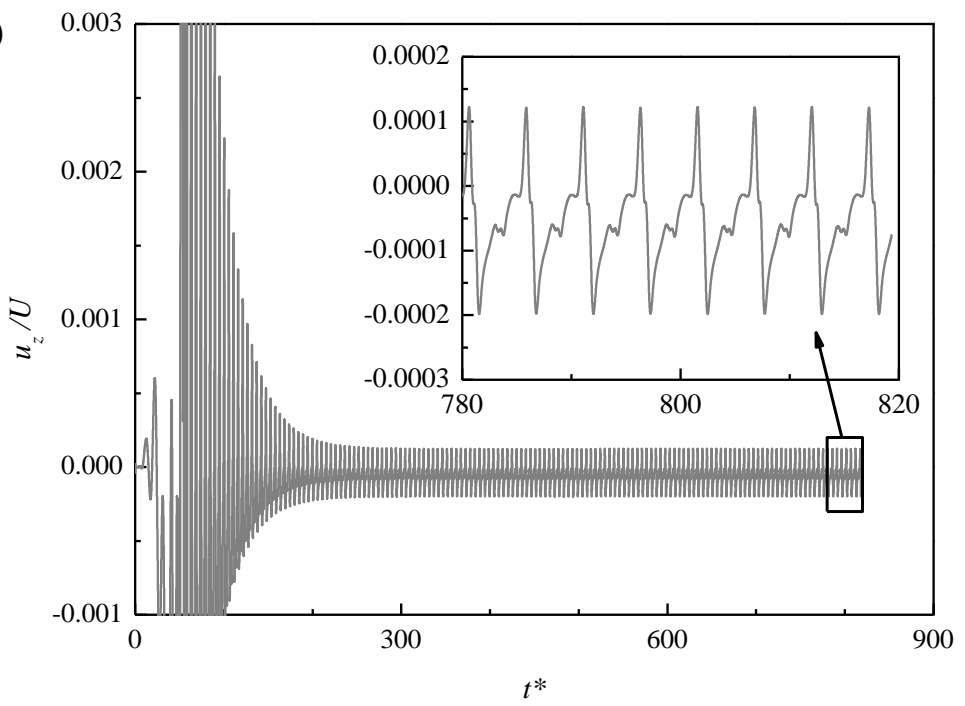

(c)

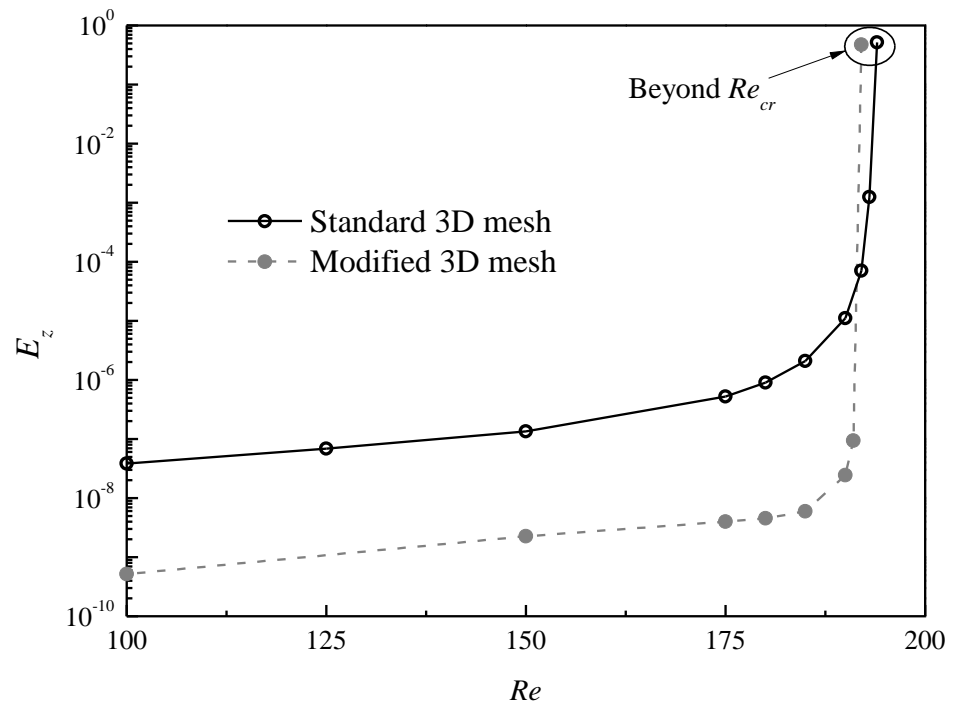

Fig. 2. (a) Time evolution of $E_{z}$ for a few cases calculated with the standard 3D mesh, (b) Time-history of the spanwise velocity sampled at $(x / D, y / D, z / D)=(3,0.5,6)$ for 
$R e=175$ with the standard 3D mesh, and (c) Variations of the statistical stationary $E_{z}$ in the saturated flow with $R e$ by using the standard and modified 3D meshes.

Fig. 2(c) shows the variations of the statistical stationary $E_{z}$ in the saturated flow with $R e$ by using the standard and modified 3D meshes. Due to the existence of small-scale disturbances, non-zero values of $E_{z}$ are detected at $R e$ well below the $R e_{c r}$. The statistical stationary $E_{z}$, although very small in magnitude, increases with $R e$ gradually for $R e<R e_{c r}$ and suddenly increases by a few orders of magnitude when $R e$ reaches $R e_{c r}$. For $R e>R e_{c r}$ and up to at least $R e=300$, the statistical stationary $E_{z}$ is saturated at a similar order of magnitude $\left(\right.$ of $10^{\circ}$ ) due to the occurrence of Mode $\mathrm{A}^{*}$ or Mode B instability of flow (Jiang et al., 2016).

Since the disturbance level in the modified mesh is significantly lower than that in the standard mesh, the magnitude of the integrated $E_{z}$ for $R e<R e_{c r}$ by using the modified mesh is approximately two orders of magnitude smaller than that based on the standard mesh (Fig. 2(c)). For $R e>R e_{c}$, however, the statistical stationary $E_{z}$ values obtained using the two meshes reach similar saturated levels. Consequently, as $R e$ approaches to $R e_{c r}$, the increase rate of $E_{z}$ predicted with the modified mesh is higher than that predicted with the standard mesh (Fig. 2(c)). Based on linear stability theory, the flow three-dimensionality developed at $R e<R e_{c r}$ would be damped out completely if the disturbances due to the skewed mesh elements could have been switched off completely, which would result in an infinite increase rate of $E_{z}$ at $R e=$ $R e_{c r}$. This is unlikely to be achieved with the present FVM model but will be investigated further with a spectral element method in $\S 3.3$.

\subsection{D flow structure for $R e<R e_{c r}$}

The wake flow structures associated with the non-zero $E_{z}$ values shown in Fig. 2(c) are examined by the iso-surfaces of the normalized streamwise vorticity $\omega_{x}$, where $\omega_{x}$ is defined as: 
$\omega_{x}=\left(\frac{\partial u_{z}}{\partial y}-\frac{\partial u_{y}}{\partial z}\right) \frac{D}{U}$

The iso-surfaces of $\omega_{x}$ obtained with the standard and modified meshes are shown in Fig. 3 and Fig. 4, respectively. The results for $R e<R e_{c r}$ are obtained from the end of each calculation, at which point the flow is fully developed (see Fig. 2(a)).

As shown in Fig. 3, for $R e=194\left(>R e_{c r}\right)$, the wake flow is initialized with the formation of a well-defined Mode A structure with a spanwise wavelength of around $4 D$ (Fig. 3(a)), followed by the natural development of a more stable pattern with large-scale vortex dislocations, i.e. Mode A* structure (Fig. 3(b)). This phenomenon has been observed by Williamson $(1992,1996 \mathrm{~b})$ through physical experiments and confirmed by numerical studies based on different mathematical formulations (e.g. Henderson, 1997; Braza et al., 2001; Behara and Mittal, 2010; Jiang et al., 2016).

For $R e<R e_{c r}$, the iso-surfaces of $\omega_{x}$ are much weaker, and the magnitude of $\omega_{x}$ reduces with decrease of $R e$ (Fig. 3), which is consistent with the trend in $E_{z}$ shown in Fig. 2(c). The well-defined wake structures for $R e=193-180$, which have a spanwise periodicity of $4 D$ and an out-of-phase sequence between the neighbouring braids, appear to be consistent with the Mode A structure (without dislocations) for $R e>R e_{c r}$ (e.g. Fig. 3(a)). As shown in Fig. 3, the wake structures for $R e<R e_{c r}$ gradually become less regular as $R e$ is decreased. The wake structures observed for $R e \leq 175$ do show some 3D features that are similar to Mode A structure, although they are not as well-defined as those observed for $R e \geq 180$.

Overall, the DNS results indicate that a fully developed wake structure for $R e<$ $R e_{c r}$ reaches to a small-amplitude stable state without large-scale vortex dislocations, whereas a regular Mode A structure for $R e>R e_{c r}$ will evolve into vortex dislocations spontaneously. The characteristics of the stable wake structure for $\operatorname{Re}<\operatorname{Re} c r$ appear to fit with the stable state of Mode A inferred by Williamson (1996b). For this reason, we refer this wake structure as stable state of Mode A structure. 
(a) $\operatorname{Re}=194, t^{*}=1900,\left|\omega_{x}\right|=0.2$

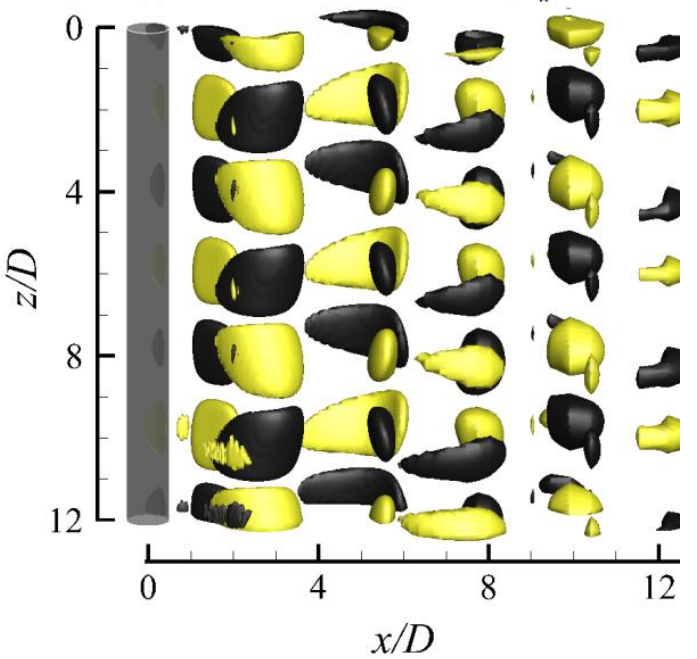

(c) $R e=193, t^{*}=5450,\left|\omega_{x}\right|=0.02$

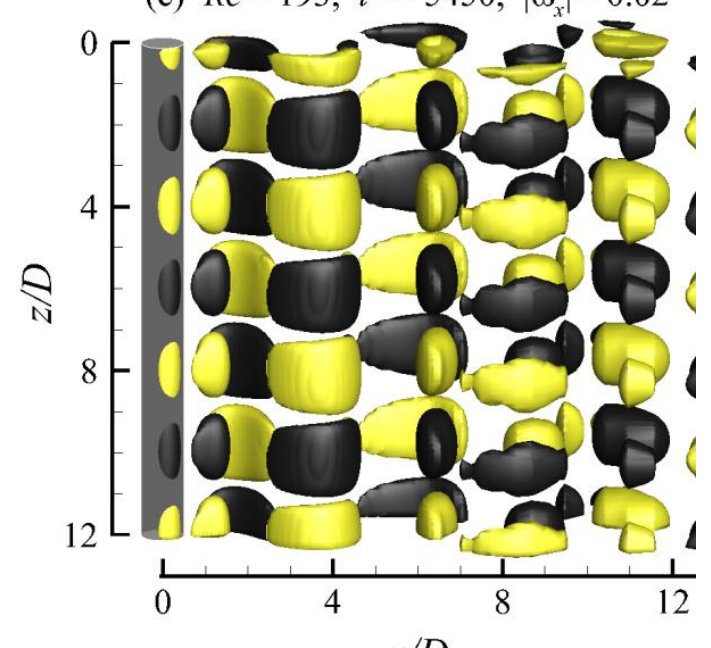

$$
x / D
$$

(e) $R e=185, t^{*}=700,\left|\omega_{x}\right|=0.001$

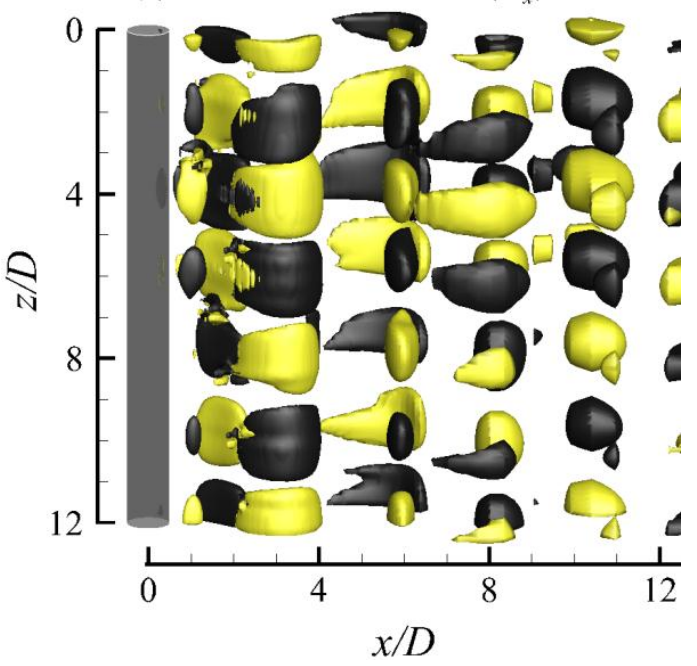

(b) $R e=194, t^{*}=3590,\left|\omega_{x}\right|=0.5$

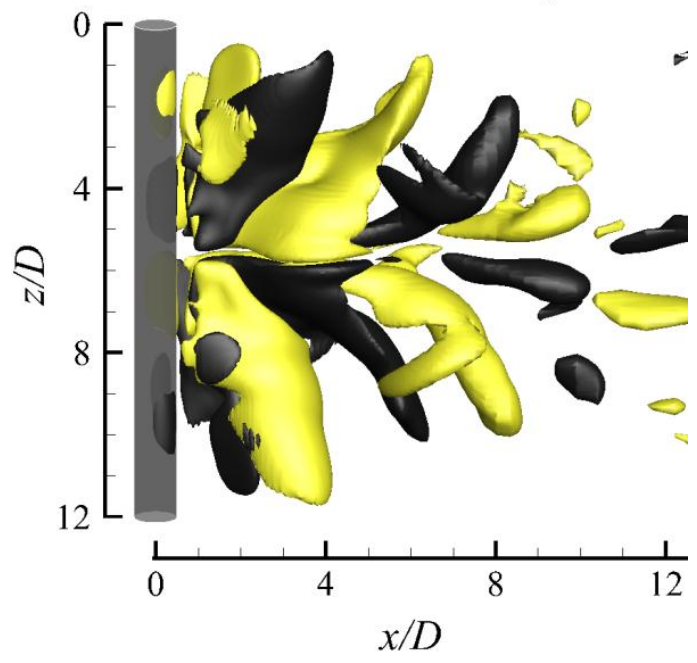

(d) $R e=190, t^{*}=1200,\left|\omega_{x}\right|=0.002$

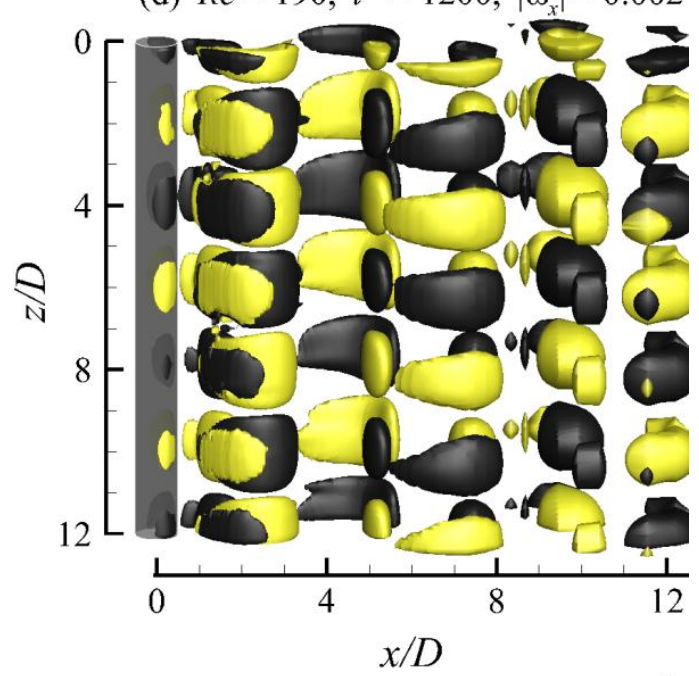

(f) $R e=180, t^{*}=740,\left|\omega_{x}\right|=8 \times 10^{-4}$

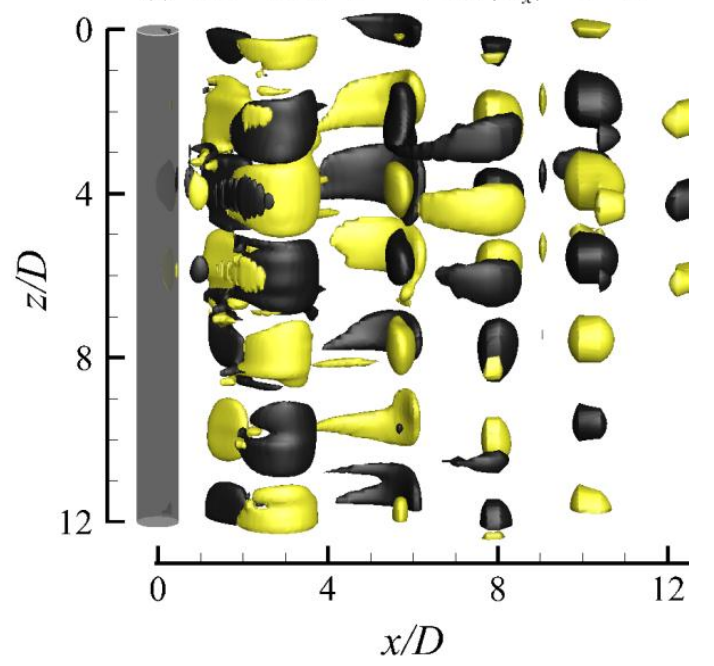


(g) $R e=175, t^{*}=810,\left|\omega_{x}\right|=7 \times 10^{-4}$

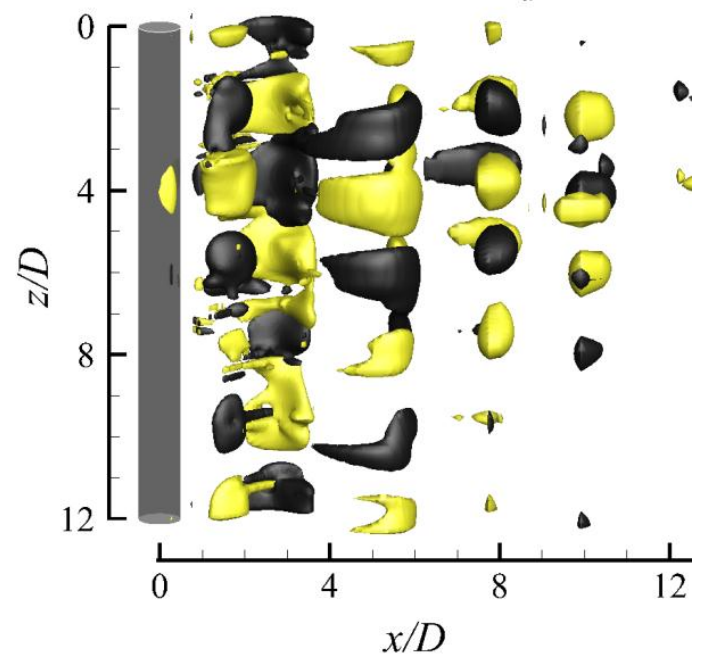

(h) $R e=150, t^{*}=570,\left|\omega_{x}\right|=4 \times 10^{-4}$

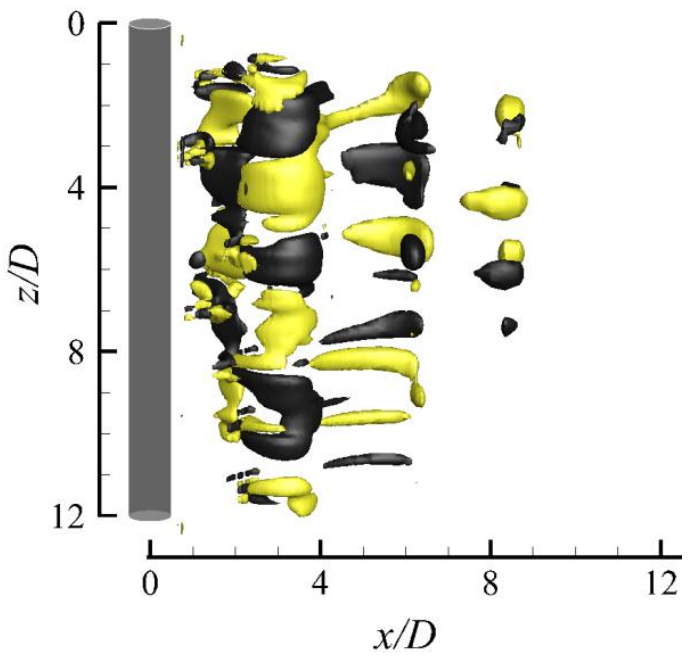

Fig. 3. Iso-surfaces of $\left|\omega_{x}\right|$ for various Re values obtained with the standard 3D mesh. The iso-surfaces are shown in different $\left|\omega_{x}\right|$ values for different $R e$ so as to display the main features of the wake structures. Dark grey and light yellow denote positive and negative values, respectively. The flow is from the left to the right past the cylinder on the left.

The fully developed wake structures obtained with the modified mesh are visualized by the iso-surfaces of $\omega_{x}$ shown in Fig. 4. At $R e=191$, which is just below the $R e_{c r}$, well-defined stable state of Mode A structure similar to that observed in Fig. 3 is observed in Fig. 4(a). Due to the decrease of the disturbance level by using the modified mesh with respect to the standard mesh, the magnitude of $\omega_{x}$ is 1 to 2 orders smaller than that observed with the standard mesh, which is consistent with the results of the integrated $E_{z}$ value shown in Fig. 2(c). For $R e \leq 185$, the well-defined stable state of Mode A structure as shown in Fig. 4(a) is not developed (Fig. 4(b)). This is attributed to the significant reduction of the disturbance level in the modified mesh. 
(a) $R e=191, t^{*}=2400,\left|\omega_{x}\right|=2 \times 10^{-4}$

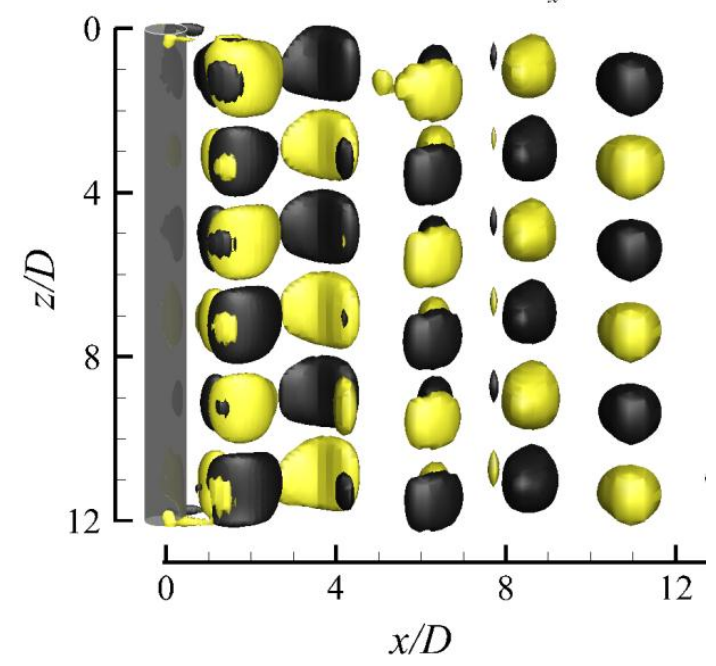

(b) $R e=185, t^{*}=400,\left|\omega_{x}\right|=3 \times 10^{-5}$

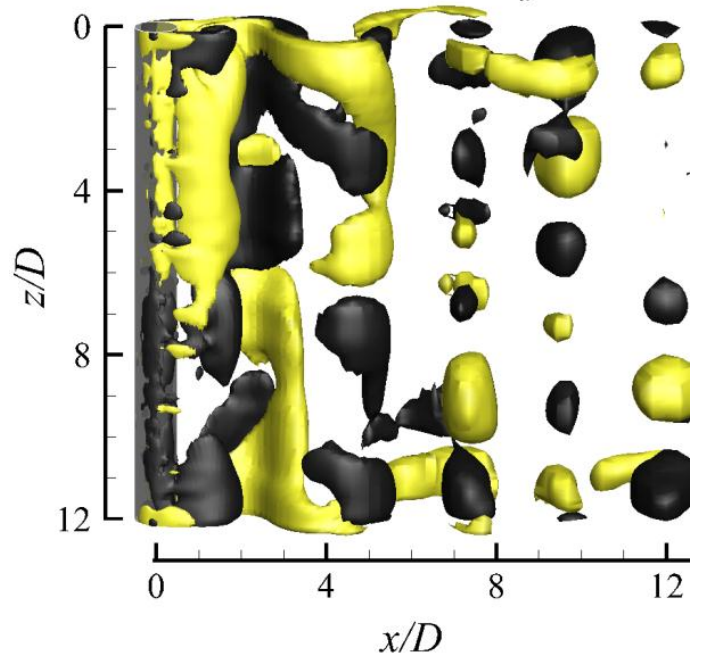

Fig. 4. Iso-surfaces of $\left|\omega_{x}\right|$ for various $R e$ values obtained with the modified 3D mesh. The iso-surfaces are shown in different $\left|\omega_{x}\right|$ values for different $R e$ so as to display the main features of the wake structures. Dark grey and light yellow denote positive and negative values, respectively. The flow is from the left to the right past the cylinder on the left.

By comparing the results shown in Fig. 3 and Fig. 4, it is found that the emergence of stable state of Mode A structure is correlated with the amplitude of the persistent disturbance introduced in the computational domain. When the disturbance level is increased, a stable state of Mode A structure will emerge at a lower Re. This is further confirmed by simulations with the modified 3D mesh where additional artificial disturbance is deliberately introduced in a small region at the front of the cylinder. The disturbance region, as shown schematically in Fig. 5 in the $x-y$ plane, is expressed as $0.5<r / D \leq 0.6,-9.5^{\circ} \leq \theta \leq 9.5^{\circ}$, and $0 \leq z / D \leq 12$ (where $r$ is the radial distance from the cylinder centre and $\theta$ is the polar angle from the front point of the cylinder). A random spanwise disturbance with an amplitude of $A_{0}=5 \times 10^{-3} U$ is maintained in this region through switching on the disturbance in the region whenever the averaged spanwise velocity in the region falls below $0.25 A_{0}$, such that the disturbance level in the neighbourhood of the region will not accumulate with time. The spanwise disturbance applied to each of the cells in the disturbance region is expressed as: 
$\left(\frac{u_{z}}{U}\right)_{i}=2(\operatorname{rand}(0,1)-0.5) \frac{A_{0}}{U} \quad$ when $\frac{\int_{V}\left|u_{z} / U\right| \mathrm{d} V}{V}<\frac{0.25 A_{0}}{U}$

where rand $(0,1)$ each time generates a random number between 0 and 1.

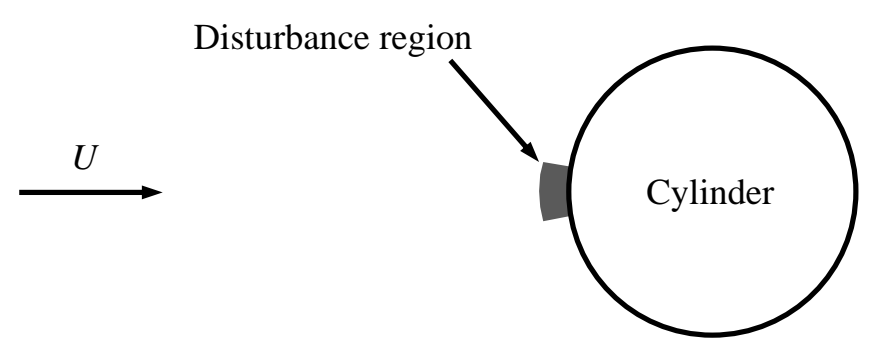

Fig. 5. Schematic model showing the region at the front of the cylinder for the introduction of additional spanwise disturbance.

Fig. 6 shows the iso-surfaces of $\omega_{x}$ obtained with the modified mesh with additional artificial disturbance introduced at the front of the cylinder. Compared with the wake structures shown in Fig. 4, additional artificial disturbance indeed triggers stable state of Mode A structure at $R e$ values lower than that for the case without such disturbance. It is also seen in Fig. 6 that the stable state of Mode A structure gradually becomes less well-defined as $R e$ is decreased, which is similar to the trend shown in Fig. 3.
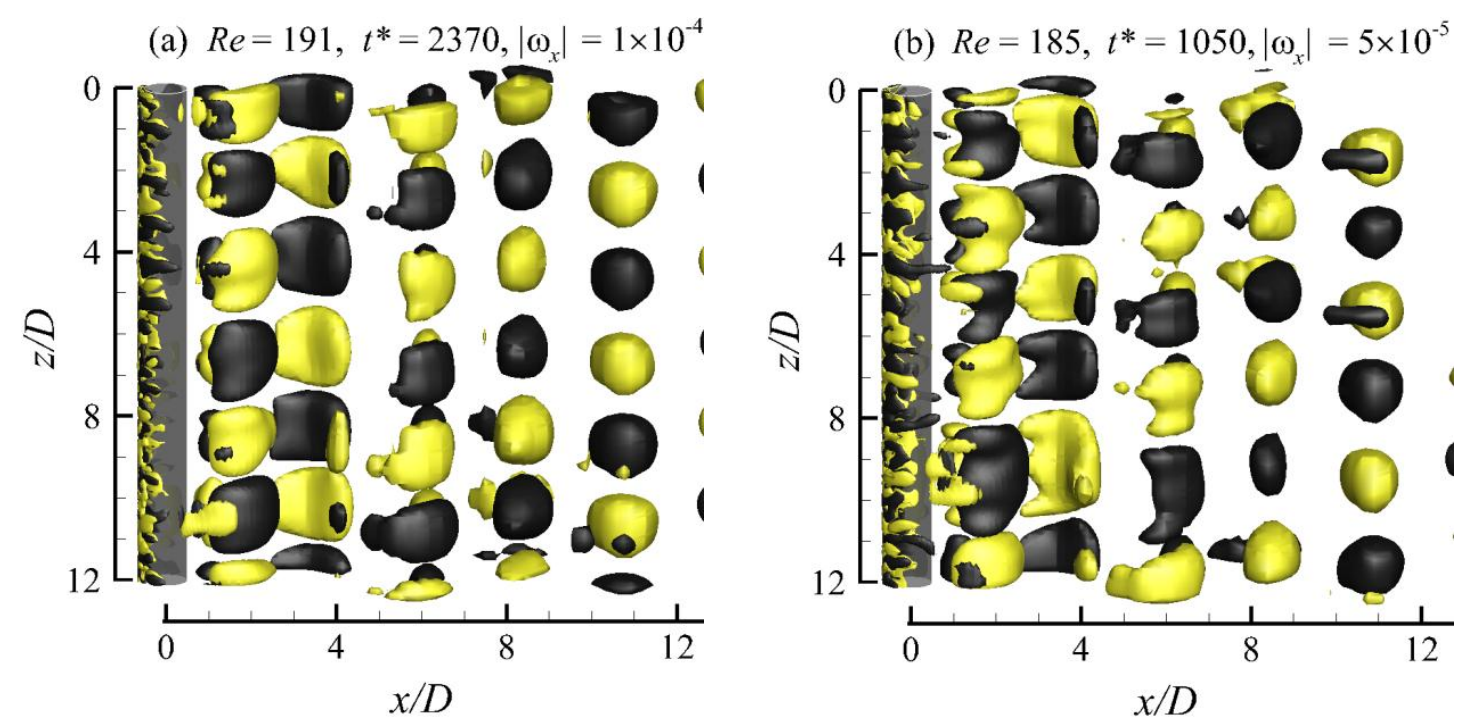
(c) $R e=180, t^{*}=1300,\left|\omega_{x}\right|=5 \times 10^{-5}$

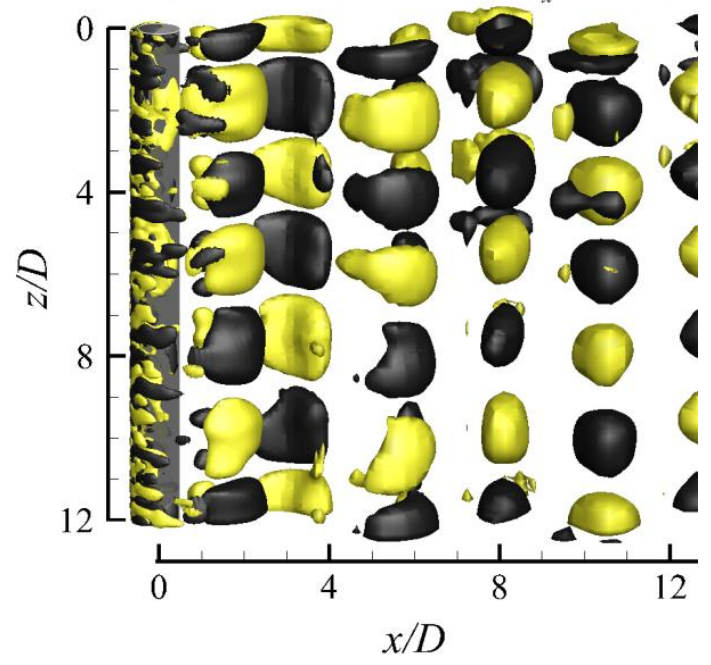

(d) $R e=175, t^{*}=880,\left|\omega_{r}\right|=4 \times 10^{-5}$

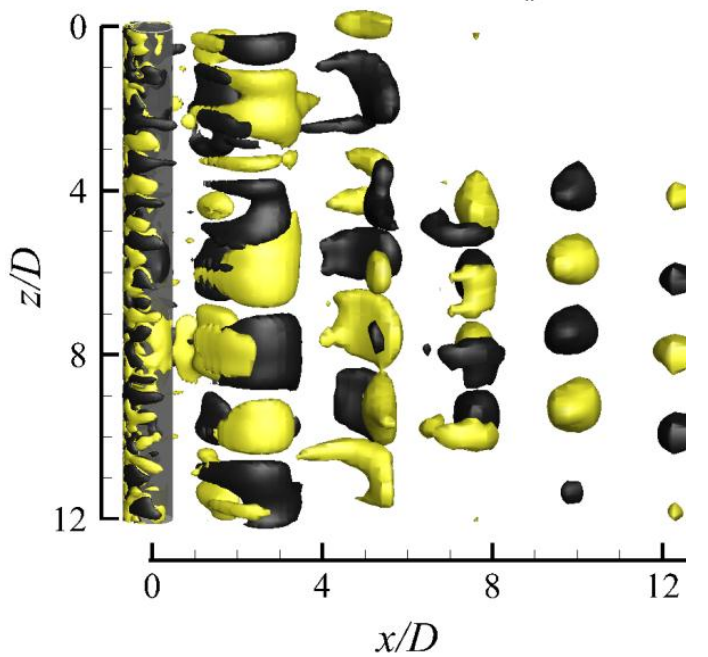

Fig. 6. Iso-surfaces of $\left|\omega_{x}\right|$ for various $R e$ values obtained with the modified 3D mesh with additional artificial disturbance introduced in a small region at the front of the cylinder. The iso-surfaces are shown in different $\left|\omega_{x}\right|$ values for different $R e$ so as to display the main features of the wake structures. Dark grey and light yellow denote positive and negative values, respectively. The flow is from the left to the right past the cylinder on the left.

\subsection{Confirmation with spectral element method}

To confirm and investigate the stable state of Mode A structure further, 3D DNS has also been carried out using a spectral element method at various $R e$ values ranging from 150 to 200 . The spectral element model used in this study is based on the framework of Cantwell et al. (2015) and will not be further detailed here. The computational domain is discretized by spectral/hp elements in the $x-y$ plane and a Galerkin Fourier expansion in the spanwise direction. It has been checked that the simulation results are relatively independent of the computational domain size $(50 D \times 40 D \times 12 D)$, order of the polynomial (=9), and the number of modes in the Fourier expansion $(=64)$. A random disturbance equal to $1 \%$ of the incoming flow velocity in all three directions is applied at the inlet of computational domain in the first time step of the simulation and is switched off immediately after the first time step. The 3D DNS results obtained using the spectral element method are summarized below: 
(1) The $R e_{c r}$ predicted by the spectral element method is approximately 189 , which is consistent with the prediction results using linear stability analysis (e.g. Barkley and Henderson, 1996).

(2) At initial stages of the simulations for $175 \leq R e<R e_{c r}$, a well-defined stable state of Mode A structure which is similar to that observed in Fig. 3(c) and Fig. 4(a) is observed by using the spectral element method (Fig. 7). For $R e \leq 170$, however, the wake structure during the initial stages of simulations (e.g. Fig. 7(g)) is similar to that shown in Fig. 4(b), for which the regular stable state of Mode A structure is not well-developed.

(3) As shown in Fig. 7, the flow three-dimensionality (represented by the stable state of Mode A structure) observed at $R e<R e_{c r}$ is transient and will be damped out eventually when the simulation is run for a sufficiently long time. For example, the maximum value of $\left|\omega_{x}\right|$ for $R e=175$ decays from $5.0 \times 10^{-3}$ at $t^{*}=200$ to $1.7 \times 10^{-6}$ at $t^{*}=500$.

(a) $R e=187, t^{*}=200,\left|\omega_{x}\right|=0.07$

(b) $R e=187, t^{*}=350,\left|\omega_{x}\right|=0.07$
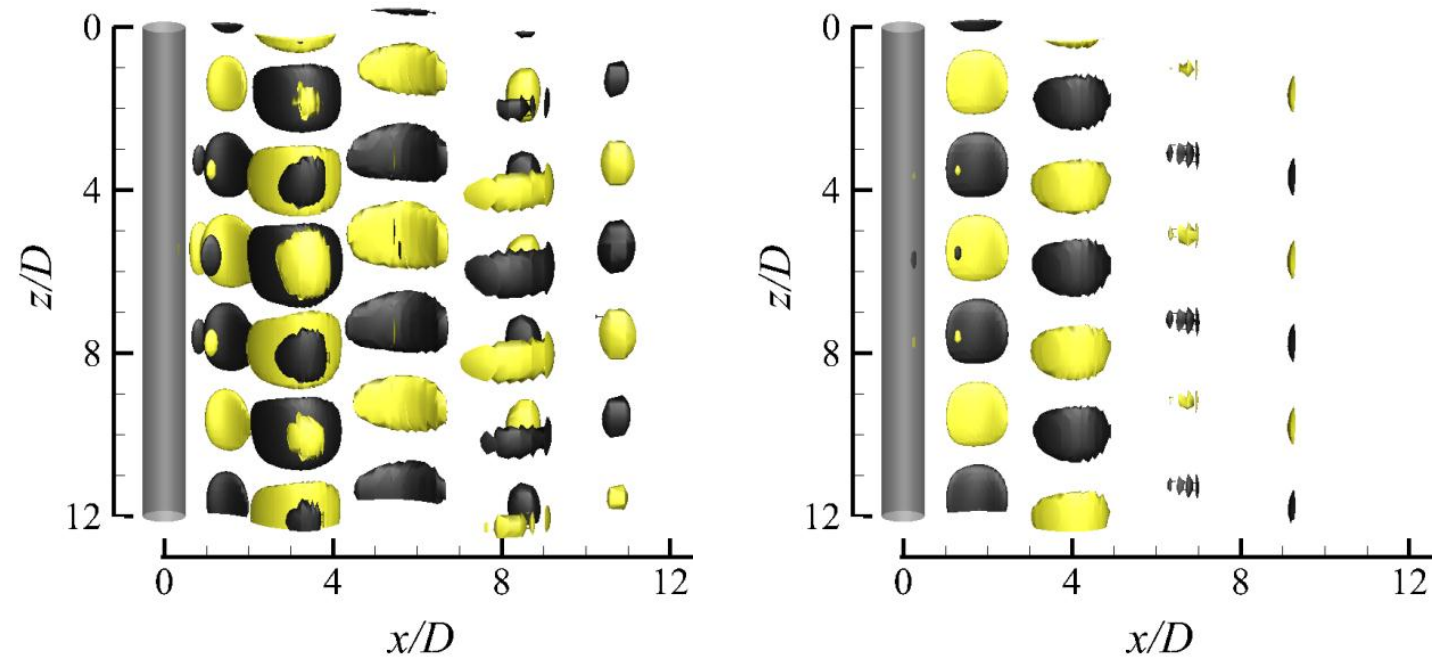

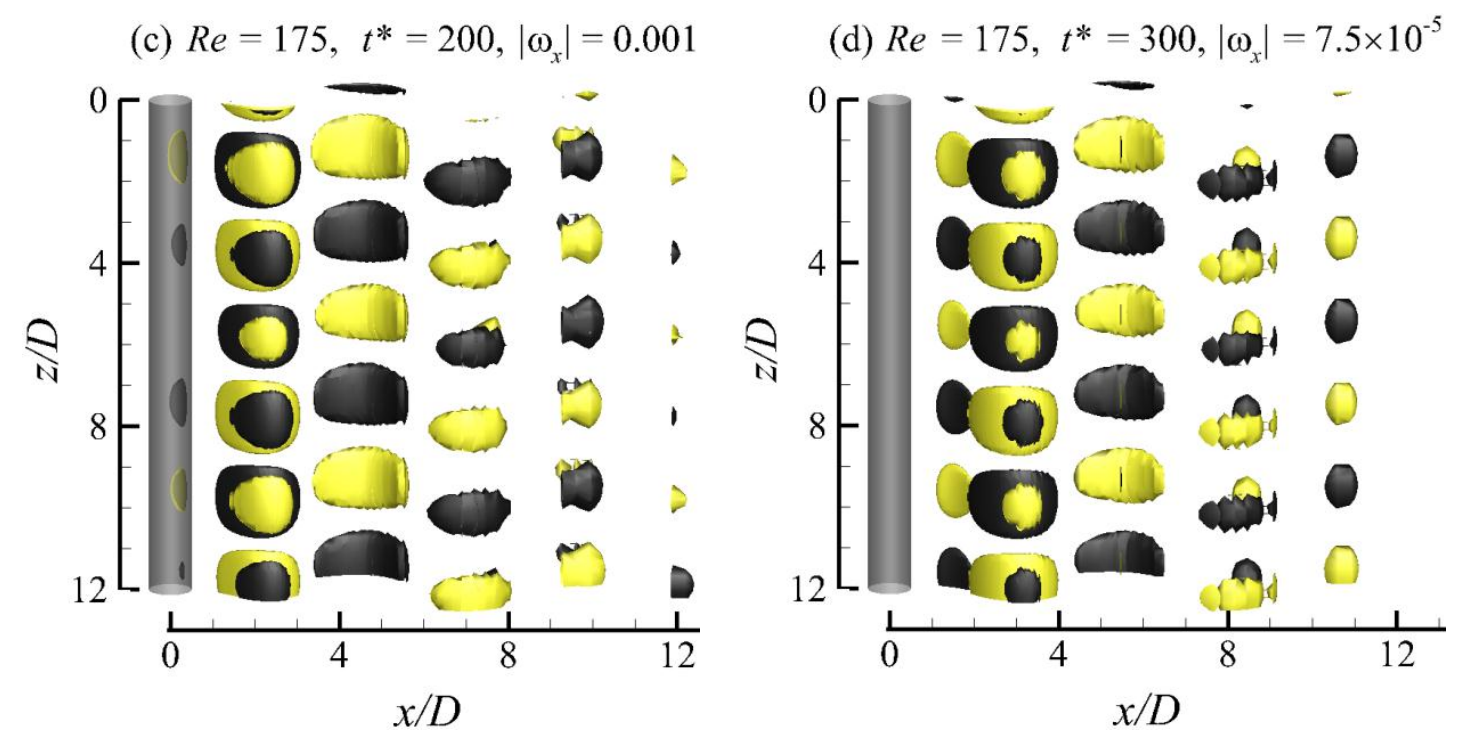

(e) $\operatorname{Re}=175, t^{*}=400,\left|\omega_{x}\right|=5 \times 10^{-6}$

(f) $R e=175, t^{*}=500,\left|\omega_{x}\right|=2.5 \times 10^{-7}$
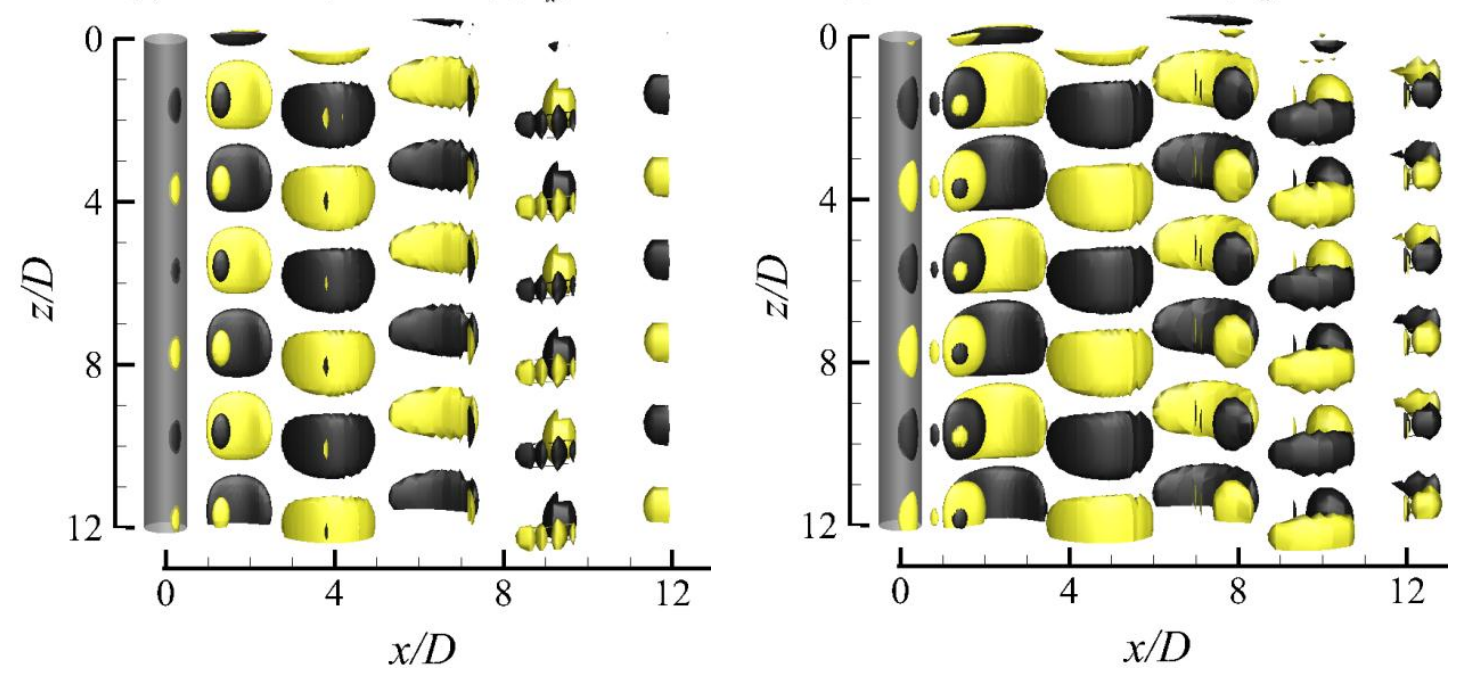

(g) $R e=170, t^{*}=310,\left|\omega_{x}\right|=2 \times 10^{-6}$

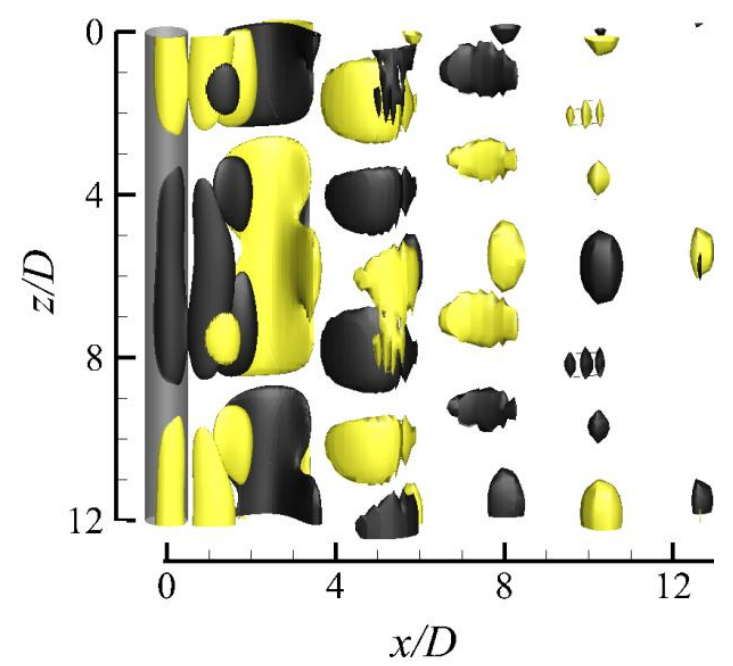

Fig. 7. Iso-surfaces of $\left|\omega_{x}\right|$ for various $R e$ values obtained using the spectral element method. The iso-surfaces are shown in different $\left|\omega_{x}\right|$ values for different $R e$ so as to 
display the main features of the wake structures. Dark grey and light yellow denote positive and negative values, respectively. The flow is from the left to the right past the cylinder on the left.

\subsection{Physical mechanism for stable state of Mode A}

The physical mechanism responsible for the stable state of Mode A wake structure observed at $R e<R e_{c r}$ is believed to be due to the convective instability of the flow, which amplifies the small-scale spanwise disturbance introduced in the computational domain upstream of the cylinder. The transient stability analysis of flow past a circular cylinder by Abdessemed et al. (2009) suggests that the energy amplification of spanwise disturbance due to convective instability could be as high as $10^{3}$ at $R e=200$ over a few vortex shedding cycles. Abdessemed et al. (2009) also found that the transient growth of initial disturbance occurs at $R e$ values much smaller than $R e_{c r}$ and that the energy amplification generally increases with increase of $R e$. When disturbance is introduced as an initial condition at $R e<R e_{c r}$, the transient flow structure formed due to the initial disturbance is expected to be eventually damped out at a rate dictated by the eigenmode (Abdessemed et al. 2009).

In the present study, the stable state of Mode A structure at $R e<R e_{c r}$ observed by the spectral element method follows the characteristics of convective instability of flow addressed by Abdessemed et al. (2009). For instance, the stable state of Mode A structure is damped out eventually in the spectral element method simulations because the disturbance is only introduced as an initial condition. In contrast, since the disturbance introduced by the FVM model (mainly due to the skewed mesh elements) is persistent throughout the simulation, it leads to a persistent energy amplification of the disturbance through convective instability of the flow, and is thus responsible for sustained stable state of Mode A structure observed at $R e<R e_{c r}$.

The present numerical results show that small-scale transient or persistent disturbance in the immediate neighbourhood of the cylinder is able to trigger the formation of well-defined stable state of Mode A structure in the near wake region at 
$R e<R e_{c r}$. Such a condition could occur in real life situations where small-scale disturbance exists throughout (e.g. through cylinder roughness or disturbance in the approaching flow). However, it is admitted that the stable state of Mode A structure discovered in this study is difficult to be captured through physical experiments due to its small amplitude. It is seen from Fig. 2(c) that the spanwise energy remains at extremely small levels until $R e$ approaches to $R e_{c r}$. Such small values of spanwise velocity may be within the range of experimental noise in most velocity measurement devices.

\subsection{Influence of disturbance level on $R e_{c r}$}

Since the bifurcation to the secondary instability is subcritical, the bifurcation point $R e_{c r}$ may be affected by the disturbance level introduced in the flow field (Barkley and Henderson, 1996). Based on the linear stability analysis results reported in the literature (e.g. Posdziech and Grundmann, 2001), the bifurcation would occur at $R e_{c r} \sim 190$ if the disturbance introduced in the flow is infinitesimal. In contrast, if the disturbance level is in the order of the free-stream velocity, the Mode $\mathrm{A}^{*}$ flow would occur and persist at an $R e$ at the lower end of the hysteresis range. According to the 3D DNS studies by Behara and Mittal (2010) and Akbar et al. (2011), the hysteresis range is about $\Delta R e=3-5$ below the $R e_{c r}$ of $\sim 190$ predicted by linear stability analysis. Within the hysteresis range, the secondary instability can only be triggered when the disturbance is beyond a certain level, and the onset point $R e_{c r}$ decreases with increase of the disturbance level (Schmid and Henningson, 2000).

Based on this, the $R e_{c r}$ for the standard and modified 3D meshes are calculated with different levels of additional artificial spanwise disturbance introduced at the front of the cylinder (the shadow region shown in Fig. 5), and the results are shown in Fig. 8. As expected, the $R e_{c r}$ decreases slightly with increase of the disturbance level due to the subcritical nature of the secondary instability. When the disturbance level is smaller than $5 \times 10^{-3} \mathrm{U}$, the influence of disturbance level on the $R e_{c r}$ is negligible.

However, with the decrease of disturbance level, the $R e_{c r}$ for the standard and 
modified 3D meshes converge at different values (Fig. 8). Excluding the influence of disturbance level, the slight difference of $R e_{c r}$ for the two meshes is believed to be attributed to the slight change of the computational mesh resolution (which would affect numerical diffusion) close to the cylinder in the $x-y$ plane due to the use of different mesh topologies (Fig. 1(b) versus Fig. 1(c)).

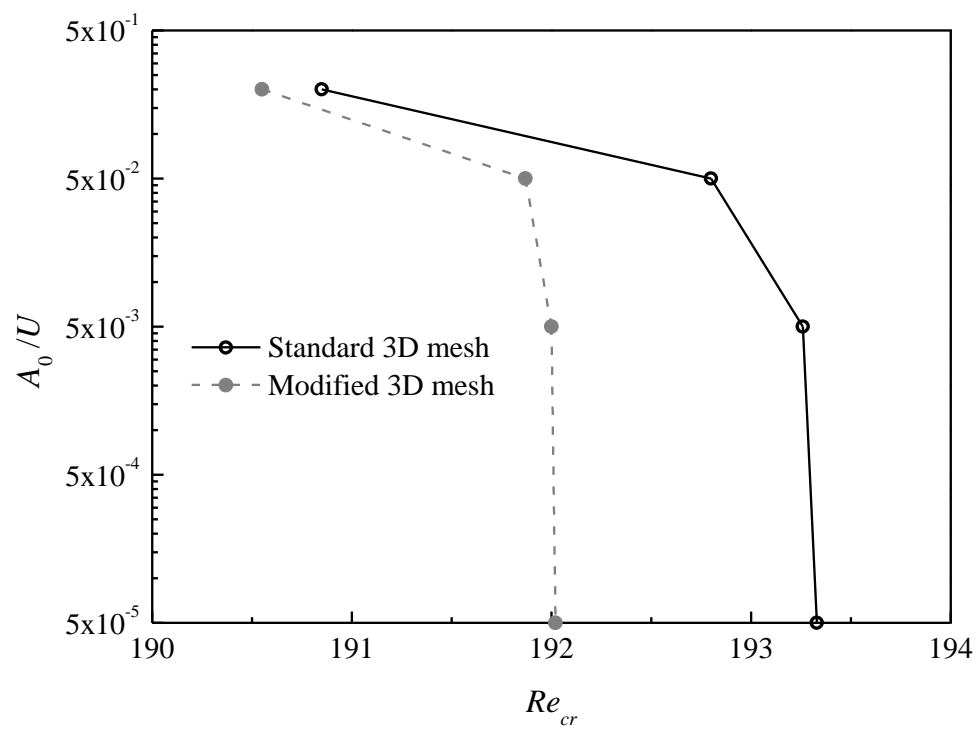

Fig. 8. Variation of the predicted $R e_{c r}$ with the level of additional artificial spanwise disturbance at the front of the cylinder.

\section{Conclusions}

This paper demonstrates the existence of stable state of Mode A wake structure for flow past a circular cylinder as inferred by Williamson (1996b), based on three-dimensional (3D) direct numerical simulations (DNS) using both finite volume and spectral element methods. The stable state of Mode A wake structure is observed at the Reynolds number $(R e)$ in a range below the critical $R e$ for Mode A* instability $\left(R e_{c r}\right)$, and this leads to a wake transition sequence of $2 \mathrm{D} \rightarrow \mathrm{A} \rightarrow \mathrm{A}^{*} \rightarrow \mathrm{B}$ as suggested by Williamson (1996b). Similar to the conventional Mode A structure observed for $R e>R e_{c r}$, the stable state of Mode A structure also has a spanwise periodicity of approximately 4 cylinder diameters and an out-of-phase sequence between the neighbouring braids. However, the stable state of Mode A structure has much weaker 
amplitude and does not evolve into large-scale vortex dislocations. This may be the reason why it could not be captured easily through physical experiments.

It is believed that the stable state of Mode A wake structure formed immediately downstream of the cylinder at $R e<R e_{c r}$ is triggered by small-scale spanwise disturbance introduced upstream of the cylinder, due to energy amplification through convective instability of the flow. Under a transient initial disturbance condition (as may be implemented using a well-refined spectral element method), the stable state of Mode A structure is transient and is damped out eventually. Under a persistent disturbance condition (as is likely to be the case using the finite volume method due to unavoidable numerical disturbance associated with a 3D mesh, or in real life situations where small-scale disturbance exists throughout), the stable state of Mode A structure is sustained throughout.

It is also found that the emergence of a stable state of Mode A structure is correlated with both $R e$ and the disturbance level. With the decrease of $R e$, the stable state of Mode A structure gradually becomes less well-defined and eventually disappears. With the decrease of the disturbance level, the stable state of Mode A structure emerges at a higher $R e$.

\section{Acknowledgments}

This work was supported by resources provided by the Pawsey Supercomputing Centre with funding from the Australian Government and the Government of Western Australia. The first author would like to acknowledge the support from the Australian Government and the University of Western Australia by providing IPRS and APA scholarships for a doctoral degree, as well as the Australia-China Natural Gas Technology Postgraduate Research Scholarships from the Australian and Western Australian Governments, the North West Shelf Joint Venture Partners, and the Western Australian Energy Research Alliance. The fifth author would like to acknowledge the support from the Australian Research Council through Discovery Early Career Research Award (DE150100428). 


\section{References}

Abdessemed, N., Sharma, A.S., Sherwin, S.J., Theofilis, V., 2009. Transient growth analysis of the flow past a circular cylinder. Physics of Fluids 21, 044103.

Akbar, T., Bouchet, G., Dušek, J., 2011. Numerical investigation of the subcritical effects at the onset of three-dimensionality in the circular cylinder wake. Physics of Fluids 23, 094103.

Barkley, D., Henderson, R.D., 1996. Three-dimensional Floquet stability analysis of the wake of a circular cylinder. Journal of Fluid Mechanics 322, 215-241.

Behara, S., Mittal, S., 2010. Wake transition in flow past a circular cylinder. Physics of Fluids 22, 114104.

Braza, M., Faghani, D., Persillon, H., 2001. Successive stages and the role of natural vortex dislocations in three-dimensional wake transition. Journal of Fluid Mechanics 439, 1-41.

Cantwell, C.D., et al., 2015. Nektar++: An open-source spectral/hp element framework. Computer Physics Communications 192, 205-219.

Henderson, R.D., 1997. Nonlinear dynamics and pattern formation in turbulent wake transition. Journal of Fluid Mechanics 352, 65-112.

Issa, R.I., 1986. Solution of implicitly discretized fluid flow equations by operator-splitting. Journal of Computational Physics 62, 40-65.

Jiang, H., Cheng, L., Draper, S., An, H., Tong, F., 2016. Three-dimensional direct numerical simulation of wake transitions of a circular cylinder. Journal of Fluid Mechanics 801, $353-391$.

Karniadakis, G.E., Triantafyllou, G.S., 1992. Three-dimensional dynamics and transition to turbulence in the wake of bluff objects. Journal of Fluid Mechanics 238, 1-30.

Miller, G.D., Williamson, C.H.K., 1994. Control of three-dimensional phase dynamics in a cylinder wake. Experiments in fluids 18, 26-35.

Norberg, C., 1994. An experimental investigation of the flow around a circular cylinder: influence of aspect ratio. Journal of Fluid Mechanics 258, 287-316.

OpenFOAM. Available from www.openfoam.org.

Posdziech, O., Grundmann, R., 2001. Numerical simulation of the flow around an infinitely long 
circular cylinder in the transition regime. Theoretical and Computational Fluid Dynamics 15, $121-141$.

Rao, A., Thompson, M.C., Leweke, T., Hourigan, K., 2013. The flow past a circular cylinder translating at different heights above a wall. Journal of Fluids and Structures 41, 9-21.

Roshko, A., 1954. On the development of turbulent wakes from vortex streets. NACA report 1191.

Schmid, P.J., Henningson, D.S., 2001. Stability and Transition in Shear Flows. New York: Springer-Verlag.

Thompson, M.C., Leweke, T., Williamson, C.H.K., 2001. The physical mechanism of transition in bluff body wakes. Journal of Fluids and Structures 15, 607-616.

Tong, F., Cheng, L., Zhao, M., Zhou, T., Chen, X., 2014. The vortex shedding around four circular cylinders in an in-line square configuration. Physics of Fluids 26, 024112.

Tritton, D.J., 1959. Experiments on the flow past a circular cylinder at low Reynolds numbers. Journal of Fluid Mechanics 6, 547-567.

Williamson, C.H.K., 1988. The existence of two stages in the transition to three-dimensionality of a cylinder wake. Physics of Fluids 31, 3165-3168.

Williamson, C.H.K., 1989. Oblique and parallel modes of vortex shedding in the wake of a circular cylinder at low Reynolds numbers. Journal of Fluid Mechanics 206, 579-627.

Williamson, C.H.K., 1992. The natural and forced formation of spot-like 'vortex dislocations' in the transition of a wake. Journal of Fluid Mechanics 243, 393-441.

Williamson, C.H.K., 1996a. Vortex dynamics in the cylinder wake. Annual Review of Fluid Mechanics 28, 477-539.

Williamson, C.H.K., 1996b. Three-dimensional wake transition. Journal of Fluid Mechanics 328, $345-407$

Zhao, M., Thapa, J., Cheng, L., Zhou, T., 2013. Three-dimensional transition of vortex shedding flow around a circular cylinder at right and oblique attacks. Physics of Fluids 25, 014105. 